\title{
OUTCOME OF CATARACT SURGERY IN A SOFT EYEBALL
}

Anupama.Kakhandaki, ShivakumarMallappa, Vishma Prabhu

1. Professor. Department of Ophthalmology, Rajarajeswari Medical College and Hospital, Bangalore

2. Professor. Department of Ophthalmology, Rajarajeswari Medical College and Hospital, Bangalore

3. Post Graduate Student. Department of Ophthalmology, Rajarajeswari Medical College and Hospital, Bangalore

\section{CORRESPONDING AUTHOR:}

Dr.Anupama.Kakhandaki,

Professor, Department of Ophthalmology,

RRMCH, Bangalore-560072

E-mail: anupamadesaiblr@gmail.com

ABSTRACT: Presenting here the outcome of cataract surgery in a $40 \mathrm{yr}$ old male patient diagnosed with mature cataract with ocular hypotony. Patient was investigated and all the possible causes of hypotony were ruled out. This was followed by an uneventful cataract surgery with a good visual outcome and normal IOP.

KEYWORDS: mature cataract, ocular hypotony

INTRODUCTION: Hypotony refers to low intra ocular pressure (IOP)and is statistically defined in many sources as IOP less than $6.5 \mathrm{mmHg} .{ }^{1}$ Low IOP can adversely impact the eye in many ways including corneal decompensation, accelerated cataract formation, maculopathy and discomfort. Hypotony in a patient with mature cataract without any other ocular pathology is not a common entity. This article briefs about such a case.

CASE REPORT: A 40 year old male patient presented to the ophthalmology OPD with complaints of diminished vision in the left eye since 1 month. It was a gradual, painless, progressive loss of vision, associated with no other complaints. There was no history of redness, watering, intake of any drugs or trauma to the eye.

Ocular examination revealed a best corrected visual acuity of $6 / 6$ in his right eye and presence of perception of light in his left eye with accurate projection of rays in all four quadrants. On slit lamp examination, anterior segment of the right eye was found to be normal, while in his left eye, patient had shallow anterior chamber, with a round briskly reacting pupil, mature white cataract (fig.1)with absence of fundal glow. Intra ocular pressure was $16 \mathrm{mmHg}$ in the right eye with applanation tonometry and was not recordable in the left eye but digitally very soft.

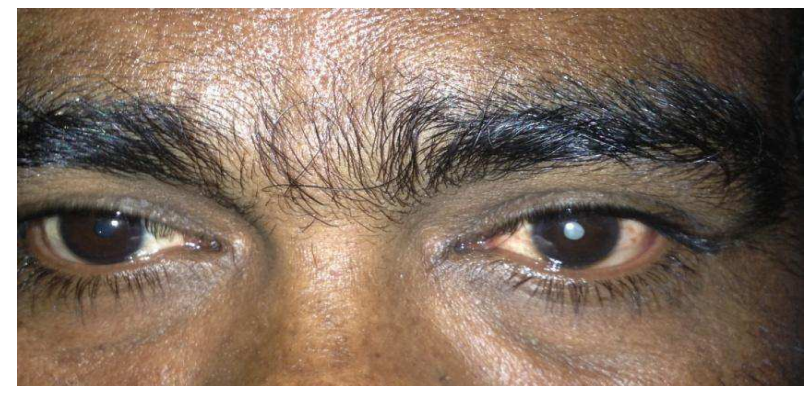

Fig.1 Shows left eye mature cataract 
B-scan of the left eye was normal except for thickening of the posterior sclera described as the ' $\mathrm{T}$ ' sign, based on this a diagnosis of posterior scleritis was made.(fig.2) The patient was started on oral Prednisolone 20mg twice a day. A CT scan of left orbit was done to confirm the findings and according to the reports it was a normal uveo-scleral coat with no evidence of retinal detachment. Routine blood investigations were normal.

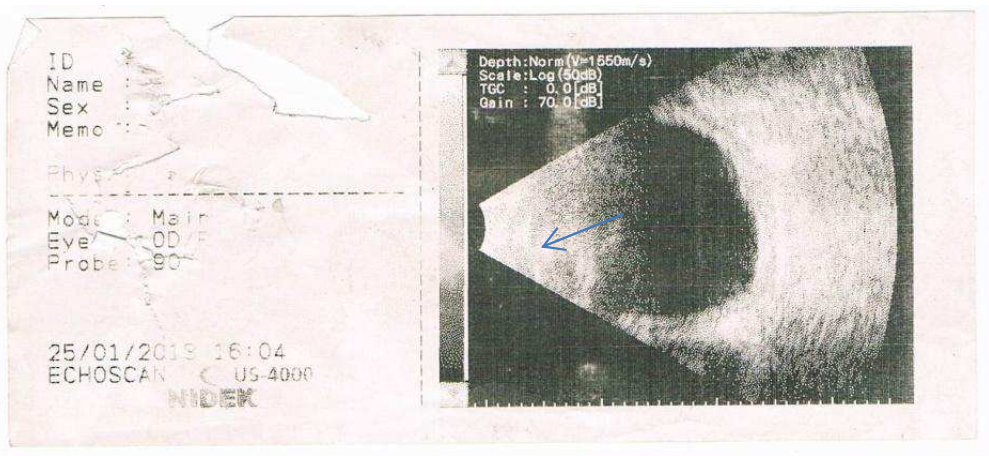

Fig.2: Shows thickening of the posterior sclera.

After 2 weeks patient was posted for left eye small incision cataract surgery with posterior chamber PMMA intraocular lens implantation. Since the IOP was not recordable prior to surgery and on the day of the surgery, it was decided that after giving local anesthetic block to the left eye, digital massage should not be given, so that the tone of the eyeball was firm. A corneoscleral frown incision was taken for better integrity of the tunnel and to prevent post operative astigmatism. Irrigating vectis was used for delivery of the nucleus (grade I). Automated irrigation and aspiration was used for cortical wash. Apart from this no other additional measures were taken in this case. It was an uneventful surgery. On the first post op day, the eye was quiet with an unaided vision of 6/9, clear cornea, well formed anterior chamber, round and briskly reactive pupil. On fundus examination, retinal folds were present in the infero-nasal quadrant while rest of the fundus was normal (fig.3). Patient had good tone in his left eye post operatively on digital tonometry. He was started on topical Gatifloxacin and Prednisolone eyedrops, 1 drop 6 times a day. Topical Ketorolac eye drops, 1 drop 4 times a day. Both the eyedrops were continued for 6 weeks with tapering of the dose of steroid eye drops to 1 drop 4 times a day after 4 weeks.

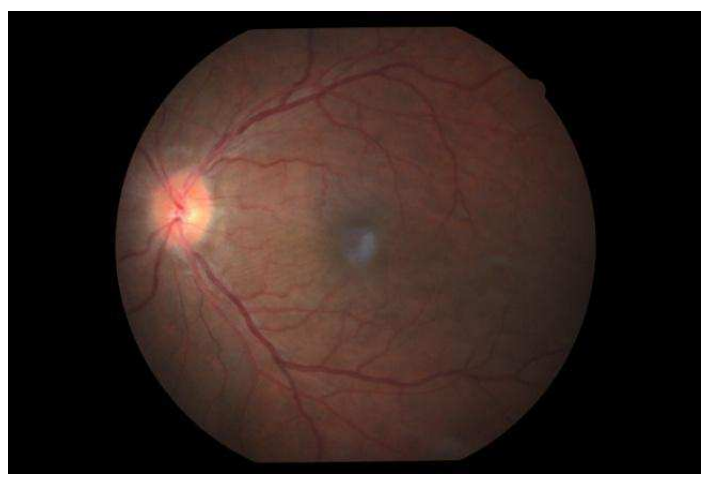

Fig.3 shows the fundus of left eye.

He was followed up every week and after 2 weeks; Oral Prednisolone, $20 \mathrm{mg}$, twice a day was stopped. There were no other changes on ocular examination. At the final follow up at 6 weeks, his left eye showed an unaided visual acuity of 6/9, quiet eye, IOP of $14 \mathrm{~mm}$ of $\mathrm{Hg}$ with 
applanation tonometer, absence of any retinal folds with a normal fundus. The B scan was normal with disappearance of the " $\mathrm{T}$ " sign in the left eye.

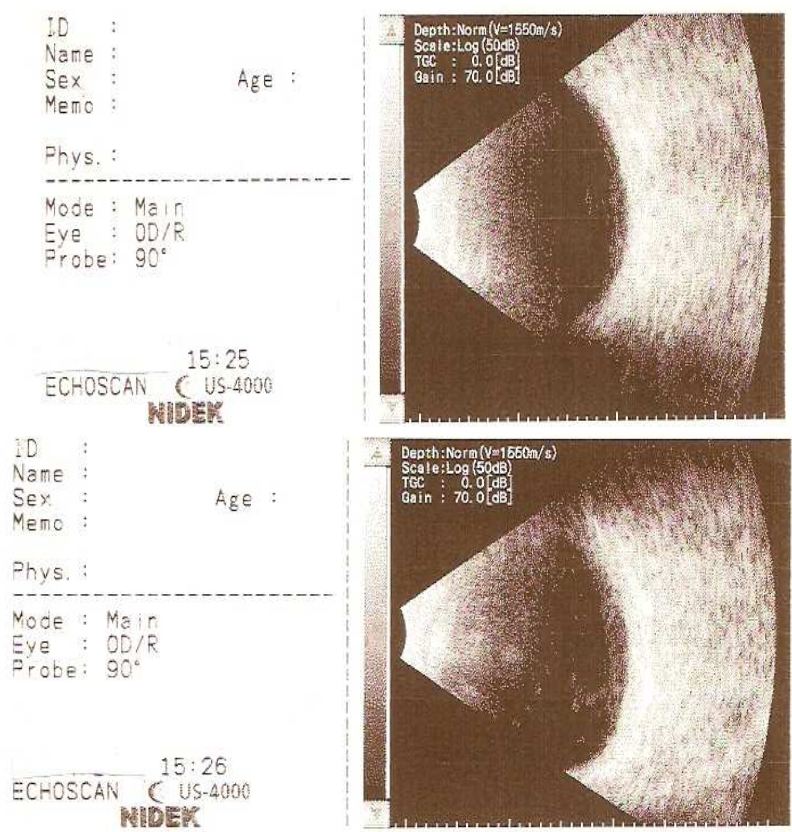

Fig- B- Scan showing disappearance of 'T' sign after 1 week of surgery.

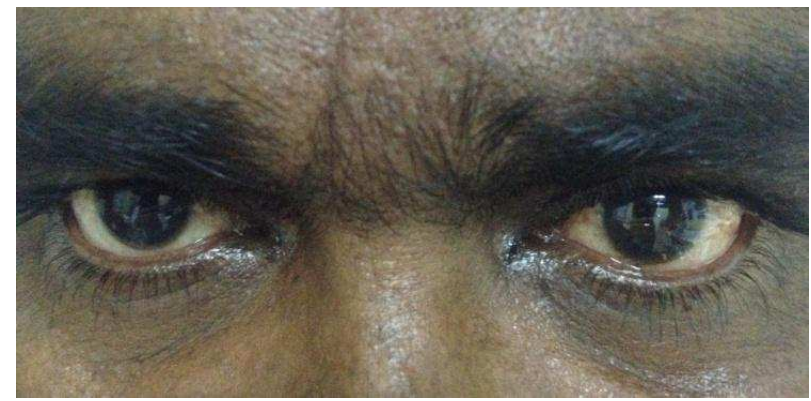

Fig. 4: shows left eye after cataract surgery.

DISCUSSION: Intra ocular pressure is a function of the rate at which aqueous humor enters the eye (in flow) and the rate at which it leaves the eye (out flow). When inflow equals outflow, a steady state exists, and the pressure remains constant. ${ }^{2}$ The control of IOP is a function of (a) production of aqueous humor (b) resistance of aqueous outflow and (c) episcleral venous pressure. ${ }^{2}$

Under normal circumstances aqueous humor is produced at the rate of $2.5 \mu \mathrm{L} /$ minute. $^{3}$ Theoretically, hypotony could result from reduced aqueous humor formation, increased unconventional outflow, or reduced episcleral venous pressure. Because the normal episcleral venous pressure is $9 \mathrm{mmHg}^{5}$, a large increase in conventional outflow facility would cause the intraocular pressure to fall toward, but not below, that level. Furthermore, the episcleral venous pressure is normal in patients with hypotony, eliminating low episcleral venous pressure as a cause of hypotony. Thus, only two possible mechanisms for hypotony exist: (1) reduced aqueous humor production and (2) increased uveoscleral outflow. 
Causes of hypotony ${ }^{1}$

a ocular surgery or trauma

b ciliochoroidal detachment

c ciliary effusion

d rhegmatogenous retinal detachment

e cyclodialysis cleft

$f$ iridocyclitis

g ocular ischemia

h traction ciliary body detachment

i pharmacological aqueous suppressants

j chemical antimetabolites

k laser or cryocyclodestruction

I Systemic hypertonicity or acidosis.

Chronic hypotony may result in several structural damages that can subsequently lead to a variety of visual symptoms. The IOP level at which structural damages arise are variable among individuals. Individuals with reduced central corneal thickness may be less likely to develop hypotony maculopathy. Increased cataract progression is seen with chronic hypotony. A shallow anterior chamber is associated with hypotony and may increase the rate of cataract formation through posterior synechiae. Also abnormal lens metabolism during hypotony, with reduced aqueous, is also thought to contribute to transient lenticular opacification and may contribute to a more gradual development of cataract. ${ }^{1}$

Several other factors may increase the likelihood of cataract progression, including preexisting cataract and administration of topical or systemic corticosteroids to treat hypotony. ${ }^{1}$

According to the study by Mackool RJ, hypotensive eye with a mature, white cataract secondary to other eye disease is not necessarily hopeless, and hypotony is not a contraindication to cataract extraction in such patients. ${ }^{4}$

CONCLUSION: Thus, hypotony alone cannot be a reason for not doing cataract surgery.

Our case highlights that inspite of pre-operative hypotony the cataract surgery was uneventful and post operative IOP stabilized. The likely explanation for ocular hypotony was long standing idiopathic posterior scleritis/intraocular inflammation. Ocular hypotony would have lead to formation of cataract.

\section{REFERENCES:}

1 Devon Ghodasra, Sandra . M. Johnson. Cataract Surgery In The Glaucoma Patient, 2009: 227-232

2 M. Bruce Shields, TEXT BOOK OF GLAUCOMA .Fourth edition. 5-25

3 Brubaker RF: The physiology of aqueous humor formation. In Drance SM, Neufeld AH (eds): Glaucoma: Applied pharmacology in medical treatment. Orlando: Grune \& Stratton, 1984:35-70

4 Mackool RJ: Mature Secondary Cataracts And Reversible Ocular Hypotension. Ophthalmic Surg 1980 Apr;11(4):256-8

5 Phelps CD, Armaly MF: Measurement of episcleral venous pressure. Am J Ophthalmol 85:35, 1978 